\title{
TooT-T: discrimination of transport proteins from non-transport proteins
}

\author{
Munira Alballa ${ }^{1 *}$ (i) and Gregory Butler ${ }^{1,2}$ \\ From Joint 30th International Conference on Genome Informatics (GIW) \& Australian Bioinformatics and Computational \\ Biology Society (ABACBS) Annual Conference
}

Sydney, Australia. 9-11 December 2019

\begin{abstract}
Background: Membrane transport proteins (transporters) play an essential role in every living cell by transporting hydrophilic molecules across the hydrophobic membranes. While the sequences of many membrane proteins are known, their structure and function is still not well characterized and understood, owing to the immense effort needed to characterize them. Therefore, there is a need for advanced computational techniques takes sequence information alone to distinguish membrane transporter proteins; this can then be used to direct new experiments and give a hint about the function of a protein.

Results: This work proposes an ensemble classifier TooT-T that is trained to optimally combine the predictions from homology annotation transfer and machine-learning methods to determine the final prediction. Experimental results obtained by cross-validation and independent testing show that combining the two approaches is more beneficial than employing only one.

Conclusion: The proposed model outperforms all of the state-of-the-art methods that rely on the protein sequence alone, with respect to accuracy and MCC. TooT-T achieved an overall accuracy of $90.07 \%$ and $92.22 \%$ and an MCC 0.80 and 0.82 with the training and independent datasets, respectively.
\end{abstract}

Keywords: Transporter prediction, Ensemble learning, Amino acid composition

\section{Background}

Membrane transport proteins control the movement of molecules across the membrane so that essential molecules such as sugars and amino acids enter the cell while waste compounds leave the cell. It is estimated that membrane transport proteins encode $2 \%$ to $16 \%$ of open reading frames in prokaryotic and eukaryotic genomes, highlighting the importance of transporters in all living species [1]. Any defective or mis-regulated membrane proteins can disturb the body's homoeostasis, thereby causing disease. Therefore, the study of cell membranes is critical for understanding the causes of many diseases and determining how to treat them. Membrane proteins

*Correspondence: m_alball@encs.concordia.ca

${ }^{1}$ Department of Computer Science and Software Engineering, Concordia University, Montréal, Québec, Canada

Full list of author information is available at the end of the article are exceptionally attractive targets for the pharmaceutical industry. Indeed, over half of today's FDA-approved drugs target them [2].

While many sequences of membrane proteins are known, due to the large number of recent genome projects, their structures and functions remain poorly characterized and understood. This is related to the immense effort necessary to characterize them because of their flexibility and instability, which creates challenges at many levels, including crystallization, expression, and structure solution. This unbalanced reality between the number of available sequences and the experimentally characterized ones has created many obstacles in the advancement of biology and drug discovery. Therefore, there is a need for advanced computational techniques that take sequence information alone to distinguish membrane transporter proteins; this can then be used to direct 
new experiments and offer clues about the function of a protein.

Earlier efforts applied homology searches of experimentally characterized databases to detect novel transporters, homology searches are still commonly used by many tools. For example, TransATH [3] (Transporters via Annotation Transfer by Homology) is a system that automates Saier's protocol based on sequence similarity. TransATH includes the computation of subcellular localization and improves the computation of transmembrane segments. The parameters of TransATH are chosen for optimal performance based on a gold standard set of transporters and non-transporters from S. cerevisiae. TransATH reports an overall accuracy of $71.0 \%$. In addition, Barghash et al. [4] annotated transporters at family and substrate levels from three organisms using sequence similarity and sequence motifs. A major limitation of homology methods, however, is that they can generate false assignments because homologous sequences do not always have significant sequence similarities. Likewise, proteins with high sequence similarities do not always share the same function [5].

More advanced methods attempt to overcome the limitations of homology methods by utilizing features from the protein sequences that better reflect the relation between the sequences and the target function. For example, TrSSP (Transporter Substrate Specificity Prediction Server) [6] is a web server for predicting membrane transport proteins and their substrate category. The TrSSP tool applies SVM in combination with the Amino Acid index (AAindex) and Position-Specific Scoring Matrix (PSSM) to predict top-level transporters and achieves a transporter prediction accuracy of $78.99 \%$ and $80.00 \%$ and a Matthews correlation coefficient (MCC) of 0.58 and 0.57 during the cross-validation and the independent testing, respectively.

SCMMTP [7] uses a novel scoring card method (SCM) that utilizes dipeptide composition to identify putative membrane transport proteins. The SCMMTP method first builds an initial matrix of 400 dipeptides and uses the difference between positive and negative compositions as an initial dipeptide scoring matrix. This matrix is then optimized using a genetic algorithm. SCMMTP achieved an overall accuracy of $81.12 \%$ and $76.11 \%$ and an MCC of 0.62 and 0.47 with the training and independent datasets, respectively.

Li et al. [8] uses SVM to predict substrate classes of transmembrane transport proteins by integrating features from PSSM, amino acid composition, biochemical properties, and Gene Ontology (GO) terms. They achieved an overall accuracy of $98.33 \%$ and an MCC of 0.97 with the independent dataset. Their method incorporates the $\mathrm{GO}$ annotation as a feature that is likely to be missing in non-annotated sequences.
Ou et al. [9] applies a word-embedding natural language processing approach to protein sequences of transporters. The protein sequence is defined as using both the word embedding and frequencies of its biological words. They achieved outstanding substrate specificity for the transporters but not for transporter detection. The accuracy for transporter prediction only reached $83.94 \%$ during the cross-validation and $85.00 \%$ with the independent datasets.

The findings from previous studies on transporter prediction can be summarized as follows: Support Vector Machine (SVM) shows superior performance compared to other machine-learning algorithms [7-9]. Moreover, the PSSM profile is a highly accurate feature for demonstrating the evolutionary information in protein sequence functional classification $[6,7,10]$.

This work focuses on distinguishing membrane transporter proteins from other non-transporter proteins. The main contributions of this work can be summarized as follows:

- We explore the practicality of using traditional homology search techniques to detect transporter proteins.

- We compare the performance of various discriminators/features on SVM models and introduce a new feature, called psi-composition, which shows superior performance to all other examined features.

- We propose a new tool, TooT-T, which employs an ensemble classifier that is trained to optimally combine the predictions obtained from homology annotation transfer and psi-composition based models to determine the final prediction. The ensemble exploits the low correlation between the predictions obtained by various methods to build a more robust classifier. The proposed model outperforms all of the state-of-the-art methods that rely on the protein sequence alone, with an overall accuracy of $90.07 \%$ and $92.22 \%$ and an MCC on 0.80 and 0.82 for the training and independent datasets respectively.

\section{Methods}

\section{Overview}

We propose an ensemble classifier that combines the results generated by two distinct methods, namely homology annotation transfer and machine learning, to detect transporter proteins. First, given a query protein $\mathrm{Q}$, a traditional homology search of the Transporter Classification Database (TCDB) is performed utilizing BLAST. A query is predicted as transporter if a hit is found using three predetermined sets of thresholds. The three predictions are delivered into the ensemble. Then, three variations of psi-composition features - psiAAC, psiPAAC, 
and psiPseAAC- are computed and input into their respective trained SVM models, the subsequent predictions are delivered to the ensemble. Finally, the trained ensemble meta-model predicts the final class as transporter $T$ or non-transporter NT. Figure 1 delineates an overview of the prediction steps. Detailed descriptions of each step are presented in the following sections.

\section{Dataset}

The same benchmarking dataset used by most transporter predictors, such as TrSSP [6], SCMMTP [7], Li et al. [8], and $\mathrm{Ou}$ et al. [9], was used to build this system.

This benchmarking dataset provided by Mishra et al. (available at http://bioinfo.noble.org/TrSSP/?dowhat= Datasets) is collected from the Swiss-Prot database. The dataset initially contained 10,780 transporter, carrier, and channel proteins that were well characterized at the protein level and had clear substrate annotations. Then, Mishra et al. removed the transporters with more than two substrate specificities, sequences with biological function annotations based solely on sequence similarity, and sequences with greater than $70 \%$ similarity. The final dataset of Mishra et al. contained a total of 1,560 sequences, divided into training and test sets, as presented in Table 1.

\section{Position specific iterated alignment compositions}

The PSI-BLAST [11] (3 iterations, e-value cutoff 0.001) search was performed on a sample protein sequence using a modified version of the Swiss-Prot database (release 2018_6) to find homologous sequences. The modified Swiss-Prot database does not include the exact hits of test sequences. Regions in the database hit sequences that were not aligned with the query protein were discarded. The query protein $(\mathrm{Q})$ and the aligned regions of its hits $\left(h_{1}, h_{2}, \ldots, h_{n}\right)$ were then used to compute position-specific iterated amino acid composition (psiAAC), pair amino acid composition (psiPAAC), and pseudo amino acid composition (psiPseAAC) as follows:

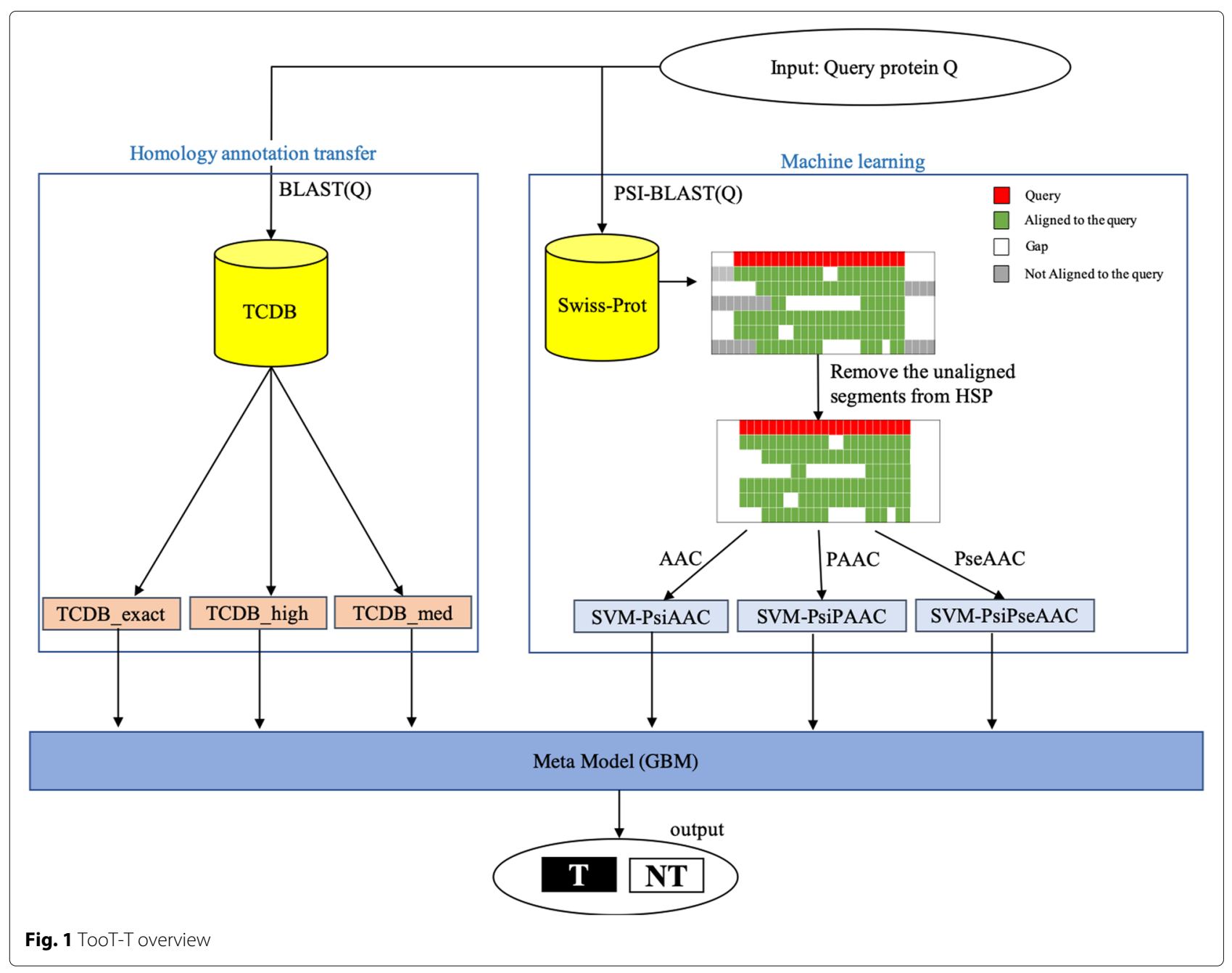


Table 1 The dataset

\begin{tabular}{lll}
\hline Class & Training dataset & Testing dataset \\
\hline Transporter & 780 & 120 \\
Non-Transporter & 600 & 60 \\
Total & 1380 & 180 \\
\hline
\end{tabular}

\section{Position Specific Iterated Amino Acid Composition (psiAAC)}

The AAC of the query protein (Q) and each of its filtered hits $\left(h_{1}, h_{2}, \ldots, h_{n}\right)$ were calculated separately as the fractions of all 20 natural amino acids and as:

$$
c_{i}=\frac{F_{i}}{L} \quad i=(1,2,3, \ldots 20)
$$

where $F_{i}$ is the frequency of the $i^{\text {th }}$ amino acid and $L$ is the length of the sequence. The AAC is represented as a vector of size 20 :

$$
A A C\left(P_{x}\right)=\left[c_{1}, c_{2}, c_{3}, \ldots, c_{20}\right] \quad x \in\left(Q, h_{1}, h_{2} \ldots, h_{n}\right)
$$

where $c_{i}$ is the composition of $i^{\text {th }}$ amino acid. The mean of individual AAC compositions represents the psi$\mathrm{AAC}$ for $\mathrm{Q}$ and was computed as:

$$
\begin{aligned}
A A C_{p s i}(Q)= & \frac{1}{n+1} \sum A A C\left(P_{x}\right) \\
& x \in\left(Q, h_{1}, h_{2} \ldots, h_{n}\right)
\end{aligned}
$$

\section{Position Specific Iterated Pair Amino Acid Composition (psiPAAC)}

Similarly, the individual PAAC descriptors for the query protein (Q) and each of its filtered hits $\left(h_{1}, h_{2}, \ldots, h_{n}\right)$ were calculated as

$$
d_{i, j}=\frac{F_{i, j}}{L-1} \quad i, j=(1,2,3, \ldots 20)
$$

where $F_{i, j}$ is the frequency of the $i^{t h}$ and $j^{t h}$ amino acids as a pair (dipeptide) and $L$ is the length of the sequence. Like AAC, PAAC is represented as a vector of size 400 , as follows:

$$
\begin{gathered}
\operatorname{PAAC}\left(P_{x}\right)=\left[d_{1,1}, d_{1,2}, d_{1,3}, \ldots, d_{20,20}\right] \\
x \in\left(Q, h_{1}, h_{2} \ldots, h_{n}\right)
\end{gathered}
$$

where $d_{i, j}$ is the dipeptide composition of the $i^{\text {th }}$ and $j^{\text {th }}$ amino acid. The mean of individual PAAC compositions represents the psiPAAC for $\mathrm{Q}$ and was computed as:

$$
\begin{aligned}
P A A C_{p s i}(Q)= & \frac{1}{n+1} \sum P A A C\left(P_{x}\right) \\
& x \in\left(Q, h_{1}, h_{2} \ldots, h_{n}\right)
\end{aligned}
$$

\section{Position Specific Iterated Pseudo Amino Acid Composition (psiPseAAC)}

The PseAAC is a combination of the 20 components of the conventional amino acid composition and a set of sequence order correlation factors that incorporates certain biochemical properties, originally proposed by Chou [12]. Given a protein sequence of length $L$ :

$$
R_{1} R_{2} R_{3} R_{4} \ldots R_{L}
$$

a set of descriptors called sequence order-correlated factors are defined as:

$$
\left\{\begin{array}{c}
\theta_{1}=\frac{1}{L-1} \sum_{i=1}^{L-1} \Theta\left(R_{i}, R_{i+1}\right) \\
\theta_{2}=\frac{1}{L-2} \sum_{i=1}^{L-2} \Theta\left(R_{i}, R_{i+2}\right) \\
\theta_{3}=\frac{1}{L-3} \sum_{i=1}^{L-3} \Theta\left(R_{i}, R_{i+3}\right) \\
\cdot \\
\cdot \\
\cdot \\
\theta_{\lambda}=\frac{1}{L-\lambda} \sum_{i=1}^{L-\lambda} \Theta\left(R_{i}, R_{i+\lambda}\right)
\end{array}\right.
$$

The parameter $\lambda$ is chosen such that $(\lambda<L)$. A correlation function is given by:

$$
\begin{aligned}
\Theta\left(R_{i}, R_{j}\right)=\frac{1}{3}\left\{\left[H_{1}\left(R_{j}\right)-H_{1}\left(R_{i}\right)\right]^{2}\right. & +\left[H_{2}\left(R_{j}\right)-H_{2}\left(R_{i}\right)\right]^{2} \\
& \left.+\left[M\left(R_{j}\right)-M\left(R_{i}\right)\right]^{2}\right\}
\end{aligned}
$$

where $H_{1}(R)$ is the hydrophobicity value, $H_{2}(R)$ is hydrophilicity value, and $M(R)$ is side chain mass of the amino acid $R_{i}$. Those quantities were converted from the original hydrophobicity, original hydrophilicity, and original side chain mass values by standard conversion as follows:

$$
H_{1}\left(R_{i}\right)=\frac{H_{1}^{\circ}\left(R_{i}\right)-\frac{1}{20} \sum_{k=1}^{20} H_{1}^{\circ}\left(R_{k}\right)}{\sqrt{\frac{\sum_{y=1}^{20}\left[H_{1}^{\circ}\left(R_{y}\right)-\frac{1}{20} \sum_{k=1}^{20} H_{1}^{\circ}\left(R_{k}\right)\right]^{2}}{20}}}
$$

where $H_{1}^{\circ}\left(R_{i}\right)$ is the original hydrophobicity value for the amino acid $R_{i}$ that was taken from Tanford [13]; $H_{2}^{\circ}\left(R_{i}\right)$ and $M^{\circ}\left(R_{i}\right)$ are converted to $H_{2}\left(R_{i}\right)$ and $M\left(R_{i}\right)$ in the same way. The original hydrophilicity value $H_{2}^{\circ}\left(R_{i}\right)$ for the amino acid $R_{i}$ was taken from Hopp and Woods [14]. The mass $M^{\circ}\left(R_{i}\right)$ of the $R_{i}$ amino acid side chain can be obtained from any biochemistry textbook. PseAAC is represented as a vector of size $(20+\lambda)$ as follows:

$$
\begin{array}{r}
\operatorname{PseAAC}\left(P_{x}\right)=\left[s_{1}, \ldots, s_{20}, s_{21}, \ldots, s_{20+\lambda}\right] \\
x \in\left(Q, h_{1}, h_{2} \ldots, h_{n}\right)
\end{array}
$$

where $s_{i}$ is the pseudo-amino acid composition such that:

$$
s_{i}=\left\{\begin{array}{lc}
\frac{f_{i}}{\sum_{r=1}^{20} f_{r}+\omega \sum_{j=1}^{\lambda} \theta_{j}} & 1 \leq i \leq 20 \\
\frac{\omega \theta_{i-20}}{\sum_{r=1}^{20} f_{r}+\omega \sum_{j=1}^{\lambda} \theta_{j}} & 20<i \leq 20+\lambda
\end{array}\right.
$$

where $f_{i}$ is the normalized occurrence frequency of the of the ith amino acid in the protein sequence, $\theta_{j}$ is the $j^{\text {th }}$ sequence order-correlated factor calculated from 
Equation 8 , and $\omega$ is a weight factor for the sequence order effect. The weight factor $\omega$ puts weight on the additional PseAAC components with respect to the conventional AAC components. The user can select any value from 0.05 to 0.7 for the weight factor. The default value given by Chou [12] is .05.

The mean of individual PseAAC compositions represents the psiPseAAC for $\mathrm{Q}$ and was computed as follows:

$$
\begin{array}{r}
\operatorname{PseAAC}_{p s i}(Q)=\frac{1}{n+1} \sum \operatorname{PseAAC}\left(P_{x}\right) \\
x \in\left(Q, h_{1}, h_{2} \ldots, h_{n}\right)
\end{array}
$$

\section{Support-vector machine}

The SVM is a powerful machine-learning tool that is used in many biological prediction tools, such as [6] and [9]. We used SVM with an RBF kernel as implemented by $\mathrm{R}$ e1071 library version 1.6-8. The best combination of $\mathrm{C}$ and $\gamma$ parameters was determined utilizing a grid-search approach.

\section{Annotation transfer by homology}

Unlike the discrete representation of a protein sample in the psi-compositions, here the protein sample was represented by its amino acid sequence and used in a similarity search-based tool (BLAST) to find similar matches in the TCDB [15]. The TCDB uses the classification system approved by the International Union of Biochemistry and Molecular Biology (IUBMB) for membrane transport proteins, known as the transporter classification (TC) system. The TCDB is a curated database of accurate and experimentally characterized transporters from over 10,000 published references. If the BLAST search produced a hit, the query was predicted to be a transporter. Since applied thresholds play an essential role in the quality of prediction, different thresholds were utilized, as shown in Table 2.

\section{Ensemble classifier}

We applied an ensemble technique known as stacked generalization, or stacking [16] to develop TooT-T. Instead of combining the predictions from multiple predictors using a simple function (such as voting), stacking trains a new model to perform the aggregation.
The stacking framework involves two levels of learning. The first level contains base-classifiers that learns directly from the training data. The second level contains a meta-classifier, that is trained using the predictions from the base-classifiers. The training instances of the meta-classifier were generated while performing the cross-validation. Algorithm 1 illustrates how the training dataset of the meta-classifier is generated [17].

\section{Algorithm 1 Stacking with $K$-fold cross validation \\ Require: Training data $\mathcal{D}=\left\{x_{i}, y_{i}\right\}\left(x_{i} \in \mathbf{R}^{n}, y_{i} \in\right.$} $\{T, N T\})$

Ensure: An ensemble classifier $H$

1: Step 1: Adopt cross validation approach in preparing a training set for meta-classifier

2: Randomly split $\mathcal{D}$ into $K$ equal-size subsets: $\mathcal{D}=$ $\left\{\mathcal{D}_{1}, \mathcal{D}_{2}, \ldots, \mathcal{D}_{K}\right\}$

3: for $k \leftarrow 1$ to $K$ do

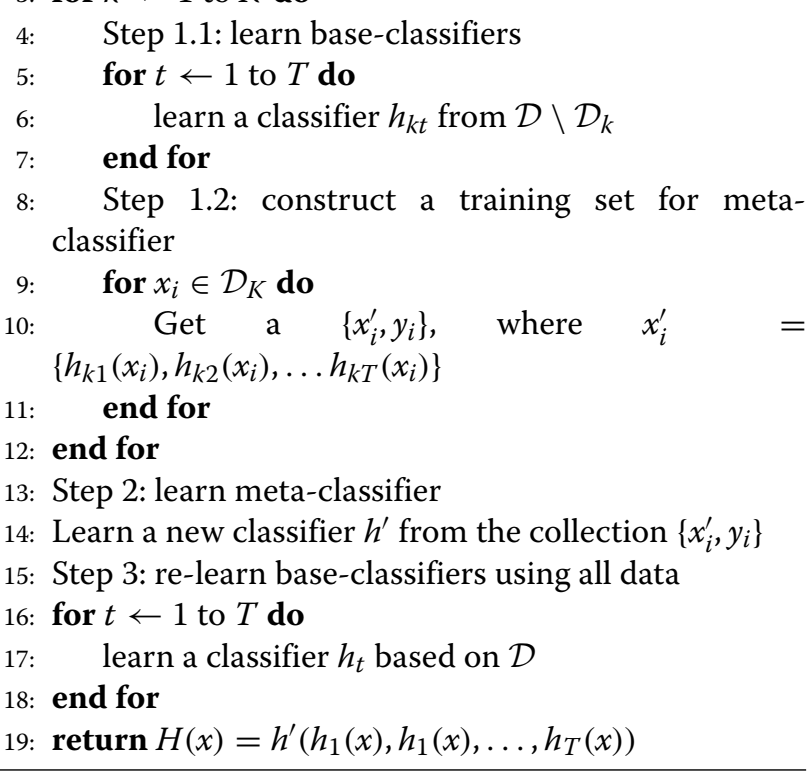

When a new query protein is input into TooT-T, the class of the query is predicted by the six base classifiers: three from SVM models that use psiAAC, psiPAAC, and psiPseAAC features respectively, and three using annotation transfer by homology utilizing different thresholds:

Table 2 Different Blast thresholds on TCDB

\begin{tabular}{lll}
\hline Name & BLAST Threshold & Motivation \\
\hline TCDB_exact & e-value=0; percent identity 100\% & exact match \\
TCDB_high & e-value 1e-20; percent identity 40\%; query & thresholds recommended by Butler et al. [3] \\
& $\begin{array}{l}\text { coverage 70\%; subject coverage 70\%; and } \\
\text { difference in length of 10\% }\end{array}$ & for TCDB Blast \\
TCDB_med & e-value 1e-8\% & threshold recommended by Barghash \\
& & et al. [4] as an acceptable normalized BLAST \\
& & threshold when dealing with a TC system \\
\end{tabular}


Table 3 Average performance of different models

\begin{tabular}{|c|c|c|c|c|c|}
\hline & Name & Sensitivity (\%) & Specificity (\%) & Accuracy (\%) & MCC \\
\hline \multirow[t]{10}{*}{ SVM } & psiPAAC* & $86.73 \pm 0.29$ & $87.99 \pm 0.54$ & $87.29 \pm 0.11$ & $0.7448 \pm 0.0027$ \\
\hline & blast-PAAC & $87.03 \pm 0.37$ & $86.08 \pm 0.24$ & $86.62 \pm 0.22$ & $0.7299 \pm 0.0045$ \\
\hline & psiAAC* & $82.69 \pm 0.21$ & $90.64 \pm 0.41$ & $86.13 \pm 0.15$ & $0.7278 \pm 0.0036$ \\
\hline & psiPseAAC* & $80.18 \pm 0.58$ & $91.51 \pm 0.45$ & $85.13 \pm 0.40$ & $0.7125 \pm 0.0075$ \\
\hline & blast-AAC & $84.97 \pm 0.35$ & $84.14 \pm 0.52$ & $84.61 \pm 0.22$ & $0.6897 \pm 0.0050$ \\
\hline & PSSM & $83.83 \pm 0.59$ & $82.03 \pm 0.59$ & $83.06 \pm 0.21$ & $0.6579 \pm 0.0038$ \\
\hline & blast-PseAAC & $84.59 \pm 0.53$ & $78.19 \pm 0.82$ & $81.81 \pm 0.35$ & $0.6306 \pm 0.0077$ \\
\hline & PseAAC & $80.45 \pm 0.42$ & $70.62 \pm 0.70$ & $76.19 \pm 0.44$ & $0.5149 \pm 0.0098$ \\
\hline & AAC & $79.73 \pm 0.50$ & $70.66 \pm 0.89$ & $75.79 \pm 0.51$ & $0.5069 \pm 0.0101$ \\
\hline & PAAC & $77.93 \pm 0.31$ & $72.14 \pm 0.56$ & $75.41 \pm 0.31$ & $0.5014 \pm 0.0062$ \\
\hline
\end{tabular}

The table shows mean $\pm s d$ performance of ten different runs of the 10-CV, in ascending order of accuracy. The asterisk symbol ${ }^{*}$ ) refers to the features used in TooT-T

TCDB_exact, TCDB_high, and TCDB_med. The six predictions are then input into the meta-classifier, which outputs the final prediction. The Gradient Boosting Machine (GBM), as implemented by caret package in R, was utilized to develop the meta-classifier.

\section{Performance evaluation}

The performance of different models was evaluated on the training dataset using 10 -fold cross-validation (10-CV), in which the training dataset was randomly partitioned into ten equally sized sets. A single set was kept as the validation data, and the remaining nine sets were used to train the respective model. The trained model was then tested using the validation set. The cross-validation process was repeated ten times, and each of the sets was used once as the validation data. The performance of each model was averaged to produce a single estimation. Since the 10-fold performance varies with different random splits, and to make the error estimation more stable, we repeated the $10-\mathrm{CV}$ ten times with different random partitions, and the performance variations between runs were captured by computing the standard deviation. It has been reported [18] that the repeated version stabilizes the error estimation, and therefore it reduces the variance of the $\mathrm{k}-\mathrm{cv}$ estimator. Throughout the rest of the paper, the crossvalidation performance is reported as mean $\pm s d$ of the ten different runs of the $10-\mathrm{CV}$.
Furthermore, the independent dataset was also used to perform a thorough evaluation experiment. The data in the independent dataset were not used during the training process and are completely unknown to our models. Four main evaluation metrics are were used to evaluate the performance: sensitivity, specificity, accuracy, and the MCC. Sensitivity, which calculates the proportion of positives (transporters) that are correctly identified.

$$
\text { Sensitivity }=\frac{T P}{T P+F N}
$$

Specificity, which measures the proportion of nontransporters that are correctly identified.

$$
\text { Specificity }=\frac{T N}{T N+F P}
$$

Accuracy, which refers to the proportion of correct predictions made divided by the total number of predictions.

$$
\text { Accuracy }=\frac{T P+T N}{T P+F N+T N+F P}
$$

The MCC is less influenced by imbalanced tests because it takes into account true and false positives and negatives. MCC values range from 1 to -1 , where 1 indicates a perfect prediction, 0 represents no better than random, and -1 implies total disagreement between prediction and observation. Higher MCC values mean that the predic-

\begin{tabular}{|c|c|c|c|c|c|c|}
\hline \multirow{2}{*}{$\begin{array}{l}\text { Encoding } \\
x\end{array}$} & \multicolumn{3}{|c|}{ Accuracy(\%) } & \multirow{2}{*}{$\begin{array}{l}\text { blast-X to X } \\
\text { increase(\%) }\end{array}$} & \multirow{2}{*}{$\begin{array}{l}\text { psi-X to } X \\
\text { increase(\%) }\end{array}$} & \multirow{2}{*}{$\begin{array}{l}\text { psi- } X \text { to blast- } X \\
\text { increase }(\%)\end{array}$} \\
\hline & $x$ & blast-X & psi-X & & & \\
\hline AAC & 75.79 & 84.61 & 86.13 & +08.82 & +10.34 & +01.52 \\
\hline PAAC & 75.41 & 86.62 & 87.29 & +11.21 & +11.88 & +00.67 \\
\hline PseAAC & 76.19 & 81.81 & 85.13 & +05.62 & +08.94 & +03.32 \\
\hline Average & 75.80 & 84.35 & 86.18 & +08.55 & +10.39 & +01.84 \\
\hline
\end{tabular}
tor has high accuracy with positive and negative classes as

Table 4 Impact of incorporating evolutionary information on the accuracy

The table notes differences in accuracy and the percentage of improvement when incorporating different evolutionary information to the baseline compositions. The highest improvement in accuracy was achieved by psi-compositions, with an average improvement of $10.39 \%$ 
Table 5 Performance of annotation transfer by homology

\begin{tabular}{|c|c|c|c|c|c|}
\hline & Name & Sensitivity (\%) & Specificity (\%) & Accuracy (\%) & MCC \\
\hline \multirow[t]{3}{*}{ ATH } & TCDB_exact & 56.92 & 95.17 & 73.55 & 0.5440 \\
\hline & TCDB_high & 85.90 & 85.50 & 85.72 & 0.7112 \\
\hline & TCDB_med & 90.38 & 64.17 & 78.98 & 0.5737 \\
\hline
\end{tabular}

The table shows the performance homology annotation transfer with the training dataset using different thresholds. The best prediction power was achieved using the TCDB_high threshold. The predicted transporter from TCDB_exact was more reliable due to its high specificity. ATH= Annotation Transfer by Homology

well as less misclassification with the two classes. MCC is considered to be the best singular assessment metric when the data are imbalanced [19-21].

$$
M C C=\frac{(T P \times T N-F P \times F N)}{\sqrt{(T P+F P) \times(T P+F N) \times(T N+F P) \times(T N+F N)}}
$$

\section{Results and discussion}

\section{Performance of transporter classification of different features}

The goal is to find the most discriminative features to represent a protein sequence, Table 3 presents the crossvalidation performance of various features on SVM models.

The examined features include: the baseline compositions where no evolutionary information is incorporated (AAC, PAAC, PseAAC), the commonly used feature to encode evolutionary information PSSM (implemented as in [6] using the same psi-composition thresholds (3 iterations, e-value cutoff 0.001)), compositions computed from sequences retrieved from the BLAST search (blast-AAC, blast-PAAC, blast-PseAAC) (e-value cutoff 0.001), and the proposed features (psiAAC, psiPAAC, psiPseAAC). Since the training data is balanced, we focus on the accuracy to evaluate the performance of different models.
The baseline compositions do not exhibit great variation in performance and have an average accuracy of $75.80 \%$. The accuracy is further boosted when evolutionary information is incorporated. While PSSM is most commonly applied in the literature to encode evolutionary information, we find that in most cases features that combine amino acid composition with evolutionary information (as described in the "Methods" section) yield higher accuracy for transporter prediction. Since the PSSM feature is also extracted from PSI-BLAST output, it is expected to show an improved performance to at least the BLASTcompositions, but this is not what is portrayed by our results. One explanation for this could be that the commonly used PSSM feature is computed from the original PSSM profile output from PSI-BLAST search to make it fixed in size $20 \times 20$. The PSSM feature, although superior to the baseline, does not capture properties to the extent shown by the amino acid composition on the returned sequences. Among all tested features, psiPAAC obtained the highest accuracy of $87.29 \%$.

The high performance of the psi-composition features is a result of incorporating two distinctive approaches, namely amino acid composition and evolutionary information. The idea is that multiple homologous sequences can reveal more about the function of a protein than a single sequence. Homologous sequences can be inferred when they share more similarity than would be expected by chance [22]. Similarity tools such as BLAST help to minimize false positives (non-homologs with significant scores; Type I errors) but do not necessarily detect remote homologs (homologs with non-significant scores; Type II errors) [22]. PSI-BLAST is more sensitive in terms of finding such remote homologs, and thus utilized by the proposed features. Furthermore, the alignment results of PSI-BLAST contains valuable information about the most conserved regions in the protein, such conservation can reflect the function of the protein. Computing

Table 6 Cross-validation performance of the proposed model

\begin{tabular}{|c|c|c|c|c|c|}
\hline & name & Sensitivity (\%) & Specificity (\%) & Accuracy (\%) & MCC \\
\hline \multirow[t]{3}{*}{ SVM } & psiAAC & $82.69 \pm 00.21$ & $90.64 \pm 00.41$ & $86.13 \pm 00.15$ & $0.7278 \pm 0.0036$ \\
\hline & psiPAAC & $86.73 \pm 00.29$ & $87.99 \pm 00.54$ & $87.29 \pm 00.11$ & $0.7448 \pm 0.0027$ \\
\hline & psiPseAAC & $80.43 \pm 00.43$ & $91.47 \pm 00.46$ & $85.23 \pm 00.34$ & $0.7142 \pm 0.0069$ \\
\hline \multirow[t]{3}{*}{ ATH } & TCDB_exact & 56.92 & 95.17 & 73.55 & 0.5440 \\
\hline & TCDB_high & 85.90 & 85.50 & 85.72 & 0.7112 \\
\hline & TCDB_med & 90.38 & 64.17 & 78.98 & 0.5737 \\
\hline \multicolumn{2}{|c|}{ Proposed_Ensemble* } & $90.15 \pm 00.24$ & $89.97 \pm 00.34$ & $90.07 \pm 00.07$ & $0.7995 \pm 0.001$ \\
\hline
\end{tabular}

The table lists the mean $\pm s d$ performance of ten different runs of the 10-CV of the proposed ensemble. It also shows the performance of each of its constituent classifiers

*The proposed model; ATH = Annotation Transfer by Homology 
Table 7 Independent testing performance of the proposed model

\begin{tabular}{ccccc}
\hline name & \multicolumn{5}{l}{ Sensitivity (\%) } & Specificity (\%) & Accuracy (\%) & MCC \\
& & & & \\
SVM psiAAC & 83.33 & 95.00 & 87.22 & 0.75 \\
psiPAAC & 89.17 & 88.33 & 88.89 & 0.76 \\
psiPseAAC & 80.00 & 96.67 & 85.56 & 0.73 \\
& & & & \\
ATH TCDB_exact & 56.67 & 91.67 & 68.33 & 0.46 \\
TCDB_high & 86.67 & 80.00 & 84.44 & 0.66 \\
TCDB_med & 92.5 & 58.33 & 81.11 & 0.56 \\
Proposed_Ensemble* & 94.17 & 88.33 & 92.22 & 0.82 \\
\hline
\end{tabular}

The table shows the performance of the proposed ensemble and each of its constituent classifiers

"The proposed model; $\mathrm{ATH}=$ Annotation Transfer by Homology

the average amino acid composition from the aligned homologous sequences thus provides a better indication of the function, and less noise, compared to computing the composition from a single sequence.

The impact of incorporating different sources of evolutionary information is presented in Table 4. The compositions computed from a single BLAST search had an average improvement from the baseline of $8.55 \%$. The psi-composition further enhanced the accuracy, with an average improvement from baseline of $10.42 \%$. The improved performance between psi-compositions and BLAST-compositions was expected because, unlike BLAST, which only uses a general scoring matrix, PSIBLAST uses a position-specific scoring matrix (PSSM) to detect sequences with a similar conservation pattern to the PSSM, thus making PSI-BLAST more sensitive to weak but biologically significant sequence relationships [11].

\section{Performance of annotation transfer by homology}

The performance of annotation transfer by homology against TCDB under different thresholds is presented in Table 5. The choice of a proper similarity threshold is critical. As shown in Table 5, there is a trade off between sensitivity and specificity, where a stricter threshold (TCDB_exact) results in low true transporter (sensitivity) detection but more reliable elimination of nontransporters (specificity). However, when the thresholds are set to be more tolerant (TCDB_med), the percentage transporter detection increases but at the cost of more false predictions. A good balance between sensitivity and specificity was achieved using thresholds suggested by [3], and the overall accuracy reached $85.72 \%$, slightly lower than the best machine-learning method psiPAAC. Nevertheless, this gives a different solution viewpoint, which we utilize in the ensemble classifier.

\section{Ensemble classifiers}

The performance of the ensemble classifier, and each of its constituent classifiers in the cross-validation and independent dataset is presented in Tables 6 and 7. The ensemble classifier consistently outperformed its classifiers in detecting transporters (sensitivity) while maintaining a credible false positive rate. Overall, it surpassed all other tested models in terms of accuracy and the MCC.

It was previously shown by $[23,24]$ that ensemble classifiers benefited the most when the individual classifiers making up the ensemble were both accurate and have low correlation (i.e., making errors in different parts of the input space). The constituent classifiers in our ensemble achieved the highest accuracy, and the correlations between them are presented in Table 8 . When combining the prediction of only the three models on the machinelearning side, we observed no improvement in overall accuracy. This is reasonable since the machine-learning models in our case were highly correlated. The obtained performance was mainly achieved by combining a different view - annotation transfer by homology, which has comparable accuracy to machine-learning classifiers but lower correlation.

\section{Comparative performance of the proposed tool with other published work}

Table 9 compares the performance of the proposed model with other published work. The highest prediction accuracy was achieved by Li et al. [8]. The high performance of their model was mainly due to using the Gene Ontology

Table 8 Pearson correlation coefficient of constituent classifiers

\begin{tabular}{lllllll}
\hline model & psiAAC & psiPAAC & psiPseAAC & TCDB_exact & TCDB_high & TCDB_med \\
\hline psiAAC & 1.00 & 0.81 & 0.90 & 0.56 & 0.63 & 0.52 \\
psiPAAC & 0.81 & 1.00 & 0.80 & 0.51 & 0.61 & 0.50 \\
psiPseAAC & 0.90 & 0.80 & 1.00 & 0.55 & 0.62 & 0.52 \\
TCDB_exact & 0.56 & 0.51 & 0.55 & 1.00 & 0.65 & 0.51 \\
TCDB_high & 0.63 & 0.61 & 0.62 & 0.65 & 0.00 & 0.78 \\
TCDB_med & 0.52 & 0.50 & 0.52 & 0.51 & 0.78 & 1.00 \\
\hline
\end{tabular}

The table shows the correlation between the constituent classifiers of the ensemble. Among themselves, the homology annotation transfer exhibit a lower correlation compared to those of the machine-learning models. This lower correlation motivates the use of ensemble techniques and helps to build a more powerful model 
Table 9 Comparison with other published work

\begin{tabular}{|c|c|c|c|c|c|c|c|c|}
\hline \multirow[t]{2}{*}{ Tool } & \multicolumn{2}{|c|}{ Sensitivity(\%) } & \multicolumn{2}{|c|}{ Specificity (\%) } & \multicolumn{2}{|c|}{ Accuracy (\%) } & \multicolumn{2}{|l|}{ MCC } \\
\hline & Ind. & $\mathrm{CV}$ & Ind. & $\mathrm{CV}$ & Ind. & $\mathrm{CV}$ & Ind. & $\mathrm{CV}$ \\
\hline SCMMTP [7] & 80.00 & 83.76 & 68.33 & 77.68 & 76.11 & 81.12 & 0.47 & 0.62 \\
\hline $\operatorname{TrSSP}[6]$ & 76.67 & 76.67 & 81.67 & 78.46 & 80.00 & 78.99 & 0.57 & 0.58 \\
\hline Ou et al. [9] & 100.00 & 83.14 & 77.50 & 84.48 & 85.00 & 83.94 & 0.73 & 0.68 \\
\hline Proposed model & 94.17 & 90.15 & 88.33 & 89.97 & 92.22 & 90.07 & 0.82 & 0.80 \\
\hline Li et al. [8] & 96.67 & 99.50 & 95.83 & 97.44 & 96.11 & 98.33 & 0.91 & 0.97 \\
\hline
\end{tabular}

(GO) annotation of the proteins as features. Such high performance is to be expected, considering the fact that all the sequences in the benchmark dataset were well annotated and extracted from the Swiss-Prot database. The goal of TooT-T is to predict novel unannotated transporters proteins.

The other tools did not incorporate annotations of proteins as features and relied solely on the protein sequence to extract features to distinguish between transporters and non-transporters. They therefore provide a better comparison with the proposed tool. Ou et al. [9] tool achieved better sensitivity (100\%) than the proposed tool (94.17\%) in the independent dataset. However, the specificity was $(77.50 \%)$ compared to $(88.33 \%)$ obtained by the proposed tool. The proposed tool achieved (7\%) higher accuracy, and (0.09) higher MCC than $\mathrm{Ou}$ et al. [9] tool in transporter detection. Overall, TooT-T achieved better accuracy, specificity, and MCC than all tools reported in all other published works, both in independent and cross-validation testing.

\section{Conclusion}

We propose an ensemble classifier that can distinguish transporter membrane proteins from other proteins. The ensemble classifier is trained to optimally combine the prediction obtained from machine-learning and homology annotation methods to produce the final prediction. The machine-learning components of the ensemble consist of SVM models that incorporate a novel feature extraction method psi-composition. The psi-composition combines traditional amino acid composition with the alignment results of PSI-BLAST and shows superior prediction performance to models built using other features, including the PSSM profile. While the prediction obtained from annotation transfer by homology was not superior to the best machine-learning models, it provided a different viewpoint on the solution. The proposed ensemble exploits the fact that different methods misclassify different sequences to build a more credible model. It was demonstrated through repeated 10 -fold cross-validation and independent dataset tests that the proposed ensemble outperformed its constituent classifiers and all other stateof-the art predictors that rely on the protein sequence alone.

\section{Abbreviations}

AAC: Amino acid composition; CV: Cross validation; GBM: Gradient boosting machine; GO: Gene ontology; IUBMB: International union of biochemistry and molecular biology; MCC: Matthews correlation coefficient; PAAC: Pair amino acid composition; PseAAC: Pseudo amino acid composition; PSSM: Position-specific scoring matrix; SCM: Scoring card method; SVM: Support-vector machine; TCDB: Transporter classification database

\section{Acknowledgments}

We thank the reviewers for their helpful and insightful comments, which have helped improve the quality of the paper.

\section{About this supplement}

This article has been published as part of BMC Bioinformatics Volume 21 Supplement 3, 2020: Proceedings of the Joint International GIW \& ABACBS-2019 Conference: bioinformatics (part 2). The full contents of the supplement are available online at https://bmcbioinformatics.biomedcentral.com/articles/ supplements/volume-21-supplement-3.

\section{Authors' contributions}

Both authors declare that they have participated in the design, implementation, and analysis of the paper, and that they have read and approved the final manuscript.

\section{Funding}

The first author is supported by King Saud University in Riyadh, Saudi Arabia, and the Saudi Arabian Cultural Bureau in Canada. The second author is supported by Natural Sciences and Engineering Research Council of Canada (NSERC), Genome Québec, and Genome Canada. Publication costs are funded by Genome Québec, and Genome Canada.

\section{Availability of data and materials}

The dataset used in the current study is publicly available at: http://bioinfo. noble.org/TrSSP/?dowhat=Datasets

\section{Ethics approval and consent to participate}

Not applicable.

\section{Consent for publication}

Not applicable.

\section{Competing interests}

The authors declare that they have no competing interests.

\section{Author details}

${ }^{1}$ Department of Computer Science and Software Engineering, Concordia University, Montréal, Québec, Canada. ${ }^{2}$ Centre for Structural and Functional Genomics, Concordia University, 24105 Montréal, Québec, Canada.

Received: 25 November 2019 Accepted: 9 December 2019 Published: 23 April 2020

\section{References}

1. Ren Q, Paulsen IT. Comparative analyses of fundamental differences in membrane transport capabilities in prokaryotes and eukaryotes. PLoS Comput Biol. 2005;1(3):27

2. Yıldırım MA, Goh K-I, Cusick ME, Barabási A-L, Vidal M. Drug-target network. Nat Biotechnol. 2007;25(10):1119. 
3. Aplop F, Butler G. TransATH: Transporter prediction via annotation transfer by homology. ARPN J Eng Appl Sci. 2017;12(2):317-24.

4. Barghash A, Helms V. Transferring functional annotations of membrane transporters on the basis of sequence similarity and sequence motifs. BMC Bioinformatics. 2013;14(1):343.

5. Whisstock JC, Lesk AM. Prediction of protein function from protein sequence and structure. Q Rev Biophys. 2003;36(03):307-40.

6. Mishra NK, Chang J, Zhao PX. Prediction of membrane transport proteins and their substrate specificities using primary sequence information. PLOS ONE. 2014;9(6):100278.

7. Liou Y-F, Vasylenko T, Yeh C-L, Lin W-C, Chiu S-H, Charoenkwan P, Shu L-S, Ho S-Y, Huang H-L. SCMMTP: identifying and characterizing membrane transport proteins using propensity scores of dipeptides. BMC Genomics. 2015;16(12):6.

8. Li L, Li J, Xiao W, Li Y, Qin Y, Zhou S, Yang H. Prediction the substrate specificities of membrane transport proteins based on support vector machine and hybrid features. IEEE/ACM Trans Comput Biol Bioinforma. 2016;13(5):947-53.

9. Ho Q-T, Phan D-V, Ou Y-Y, et al. Using word embedding technique to efficiently represent protein sequences for identifying substrate specificities of transporters. Anal Biochem. 2019;577:73-81.

10. Hu Y, Guo Y, Shi Y, Li M, PuX. A consensus subunit-specific model for annotation of substrate specificity for ABC transporters. RSC Adv. 2015;5(52):42009-19.

11. Altschul SF, Madden TL, Schäffer AA, Zhang J, Zhang Z, Miller W, Lipman DJ. Gapped BLAST and PSI-BLAST: a new generation of protein database search programs. Nucleic Acids Res. 1997;25(17):3389-402

12. Chou K-C. Prediction of protein cellular attributes using pseudo-amino acid composition. Proteins: Struct Funct Bioinforma. 2001;43(3):246-55.

13. Tanford C. Contribution of hydrophobic interactions to the stability of the globular conformation of proteins. J Am Chem Soc. 1962;84(22):4240-7.

14. Hopp TP, Woods KR. Prediction of protein antigenic determinants from amino acid sequences. Proc Natl Acad Sci. 1981;78(6):3824-8.

15. Saier Jr MH, Reddy VS, Tsu BV, Ahmed MS, Li C, Moreno-Hagelsieb G. The Transporter Classification Database (TCDB): recent advances. Nucleic Acids Res. 2015;44(D1):372-9.

16. Wolpert DH. Stacked generalization. Neural Netw. 1992;5(2):241-59.

17. Aggarwal CC. Data Classification: Algorithms and Applications; 2014 , pp. 499-500. https://doi.org/10.1201/b17320.

18. Kohavi R. Wrappers for performance enhancement and oblivious decision graphs. Technical report: Carnegie-Mellon University; 1995.

19. Ding Z. Diversified ensemble classifiers for highly imbalanced data learning and their application in bioinformatics. PhD thesis. Atlanta: Georgia State University; 2011.

20. Weiss GM, Provost F. Learning when training data are costly: The effect of class distribution on tree induction. J Artif Intell Res. 2003;19:315-54.

21. Bekkar M, Djemaa HK, Alitouche TA. Evaluation measures for models assessment over imbalanced data sets. J Inf Eng Appl. 2013;3(10):27-38.

22. Pearson WR. An introduction to sequence similarity ("homology") searching. Curr Protoc Bioinforma. 2013;42(1):3-1.

23. Opitz DW, Shavlik JW. Generating accurate and diverse members of a neural-network ensemble. In: Advances in Neural Information Processing Systems. Cambridge: MIT Press; 1996. p. 535-41.

24. Krogh A, Vedelsby J. Neural network ensembles, cross validation, and active learning. In: Advances in Neural Information Processing Systems. Cambridge: MIT Press; 1995. p. 231-8.

\section{Publisher's Note}

Springer Nature remains neutral with regard to jurisdictional claims in published maps and institutional affiliations.

Ready to submit your research? Choose BMC and benefit from:

- fast, convenient online submission

- thorough peer review by experienced researchers in your field

- rapid publication on acceptance

- support for research data, including large and complex data types

- gold Open Access which fosters wider collaboration and increased citations

- maximum visibility for your research: over $100 \mathrm{M}$ website views per year

At $\mathrm{BMC}$, research is always in progress.

Learn more biomedcentral.com/submissions 